\title{
Frecuencia y gravedad de la retinopatía del prematuro en una unidad de cuidados intensivos neonatales
}

\author{
Heladia García, Héctor González-Cabello, Carlos Augusto Soriano-Beltrán, Marco Antonio Soto-Dávila, \\ Yolanda Vázquez-Lara y Claudia Hernández-Galván \\ Instituto Mexicano del Seguro Social, Centro Médico Nacional Siglo XXI, Unidad de Investigación en Análisis y Síntesis de la Evidencia, Hospital \\ de Pediatría, Ciudad de México, México
}

\section{Resumen}

Introducción. La retinopatía del prematuro $(R O P)$ es una enfermedad en la que los vasos sanguíneos de la retina no se desarrollan normalmente, lo que puede ocasionar daño visual y ceguera. Objetivo: Identificar la frecuencia y gravedad de la ROP en recién nacidos prematuros. Método: Estudio descriptivo comparativo realizado en el periodo 2009-2013. Se registraron características generales de los pacientes, edad posnatal y edad posconcepcional al momento de la exploración oftalmológica, así como gravedad y tratamiento de la ROP. Resultados: Se incluyeron 326 recién nacidos prematuros: $47.8 \%$ ( $n=156$ ) tuvo ROP, en $21.1 \%$ fue grave (estadio $\geq 3$ ). La mediana de la edad gestacional fue de 28 semanas en los recién nacidos prematuros con ROP, el peso al nacer fue de $1000 \mathrm{~g}$ y la edad posconcepcional a la exploración oftalmológica fue de 36 semanas. De los niños con ROP, $71.1 \%$ recibió tratamiento, $63.4 \%$ de aquellos que tuvieron ROP leve y $100 \%$ de aquellos con ROP grave. Conclusiones: La frecuencia de ROP fue alta, mayor a la reportada en los países desarrollados $y$ similar a la de otros países en desarrollo. La frecuencia de ROP grave también fue mayor. Es necesario establecer programas efectivos de detección y tratamiento oportuno de ROP.

PALABRAS CLAVE: Retinopatía del prematuro. Recién nacido prematuro. Fotocoagulación.

\begin{abstract}
Introduction: Retinopathy of prematurity $(R O P)$ is a disease where retinal blood vessels do not develop normally and may cause visual damage and blindness. Objective: To determine the frequency and severity of ROP in preterm newborns. Method: A descriptive, comparative study was carried out within the 2009-2013 period. Patients' general characteristics were recorded, including gestational age and postmenstrual age at the moment of ophthalmologic examination, as well as ROP severity and type of treatment. Results: $A$ total of 326 preterm newborns were included: $47.8 \%(n=156)$ had ROP; in $21.1 \%$ it was severe (stage $\geq 3$ ). Median gestational age was 28 weeks in preterm newborns with ROP, median birth weight was $1000 \mathrm{~g}$, and median postmenstrual age at ophthalmological examination was 36 weeks. Of the infants with ROP, $71.1 \%$ received treatment: $63.4 \%$ of those who had mild ROP and $100 \%$ of those with severe ROP. Conclusions: ROP frequency was high, higher than that reported in developed countries and similar to that in developing countries. The frequency of severe ROP was also higher. It is necessary for effective programs for the detection and opportune treatment of ROP to be established.
\end{abstract}

KEY WORDS: Retinopathy of prematurity. Preterm neonate. Photocoagulation.

Correspondencia:

Heladia García

E-mail: hely1802@gmail.com
Fecha de recepción: 25-01-2018

Fecha de aceptación: 18-07-2018

DOI: 10.24875/GMM.18004145
Gac Med Mex. 2018;154:561-568

Disponible en PubMed www.gacetamedicademexico.com 


\section{Introducción}

La retinopatía del prematuro (ROP, retinopathy of prematurity) es una vitreorretinopatía fibro y vasoproliferativa periférica que se presenta en los recién nacidos (RN) prematuros sometidos a oxigenoterapia, de etiopatogenia aún desconocida. ${ }^{1}$ Fue descrita por Terry $^{2}$ en 1942 como fibroplasia retrolental. En 1951, Heath la nombró retinopatía del prematuro. ${ }^{3}$ La ROP es la causa más frecuente de ceguera infantil en países desarrollados y en desarrollo., ${ }^{1,4}$ Los avances en los cuidados intensivos neonatales han aumentado la supervivencia de los niños prematuros con peso extremadamente bajo al nacer (PNEB) y, por lo tanto, también han incrementado el número de niños con riesgo de desarrollar ROP grave. ${ }^{5}$

En la década de 1950, Kinsey ${ }^{6}$ reportó la primera epidemia de ROP y la relacionó con la oxigenoterapia, lo que llevó a restringir su uso. En la década de 1980 se presentó una segunda epidemia; ${ }^{7}$ en la actualidad se considera que está ocurriendo la tercera.

La incidencia de ROP y su modalidad grave ha mostrado diferentes tendencias. En 1956, el grupo CRYO-ROP ${ }^{8}$ reportó una incidencia de $27 \%$, mientras que en un estudio multicéntrico de 1986 y 1987 se reportó un incremento a $65.8 \%$ en RN prematuros con peso $<1251 \mathrm{~g}$ y a $81.6 \%$ en los prematuros con peso corporal < 1000 g. ${ }^{9}$ Sin embargo, entre 1995 y 1996 en este mismo grupo se reportó un descenso en la incidencia a $41.3 \%{ }^{10}$

El grupo ETROP, entre los años 2000 y 2002 reportó una incidencia de $68 \%$, similar a la reportada por el grupo CRYO- ROP en 1986 y 1987.,11

La incidencia de ROP reportada en los países desarrollados es muy variable: oscila entre 9.3 y $68 \%{ }^{12-17}$

Hay escasos informes de la incidencia de ROP en México. En los estudios realizados en diferentes centros oscila entre 13.3 y $30 \% .{ }^{18-21}$

El tamizaje para la detección precoz de la ROP se consideró prioritario a partir de $1988 .{ }^{5}$ El objetivo principal del tamizaje es identificar a todos los prematuros que requieran tratamiento para ROP o seguimiento oftalmológico más estrecho, como se especifica en las últimas recomendaciones establecidas por la Academia Americana de Pediatría, la Asociación Americana de Oftalmología Pediátrica y Estrabismo y la Academia Americana de Oftalmología. ${ }^{22}$

Las recomendaciones para el tamizaje son las siguientes: peso al nacimiento $\leq 1500 \mathrm{~g}$ o edad gestacional $\leq 32$ semanas, debe ser realizado por un oftalmólogo con experiencia en la evaluación del niño prematuro, entre las semanas cuatro y seis de edad cronológica, o entre las semanas 31 y 33 de edad posconcepcional; las evaluaciones sucesivas serán determinadas con base en los hallazgos del primer examen oftalmológico. ${ }^{22}$

Un número significativo de niños que desarrollan ROP grave tendrá resultados visuales (14.5\%) y estructurales $(9.1 \%)$ desfavorables a pesar del tratamiento ablativo con láser 0 crioterapia..$^{23,24}$

El objetivo del presente estudio fue identificar la frecuencia y gravedad de la ROP, así como el tratamiento administrado en los RN prematuros que ingresaron a la unidad de cuidados intensivos neonatales (UCIN) o que fueron atendidos en la consulta externa de oftalmología de un hospital de tercer nivel de atención.

\section{Método}

Estudio descriptivo, comparativo, de pacientes ingresados a la UCIN y a la Consulta Externa de Oftalmología del Hospital de Pediatría, Centro Médico Nacional Siglo XXI, Instituto Mexicano del Seguro Social, Ciudad de México, donde se atiende a pacientes referidos de otras unidades hospitalarias de la zona sur de la Ciudad de México, de algunos estados de la República Mexicana y de hospitales privados. El protocolo fue aprobado por el Comité Local de Investigación y Ética del hospital sede de la investigación.

Se incluyeron RN prematuros con edad gestacional al nacer $\leq 32$ semanas o peso al nacer $\leq 1500 \mathrm{~g}$, edad cronológica $\geq 4$ semanas, que recibieron oxígeno suplementario y que ingresaron a la UCIN o fueron atendidos en la consulta externa de oftalmología, en el periodo 2009-2013.

Se registraron los siguientes datos: edad gestacional, peso al nacer, sexo, calificación Apgar a los minutos 1 y 5 , vía de nacimiento, comorbilidad, uso de esteroides prenatales, surfactante exógeno y oxígeno suplementario, edad cronológica y edad posconcepcional al momento de la evaluación oftalmológica, clasificación y gravedad de la ROP y tratamiento administrado.

Se usó la clasificación internacional de la ROP, ${ }^{25}$ que la agrupa en cinco estadios y tres zonas. Además, se registró si había enfermedad plus (dilatación venosa, tortuosidad arteriolar y hemorragias en polo posterior). La gravedad se estratificó en dos categorías: ROP leve, que comprende los estadios 1 y 2, y ROP grave, que comprende los estadios 3,4 y $5 .{ }^{26}$

Los RN que cumplieron con los criterios de inclusión fueron revisados y tratados por los oftalmólogos 
Tabla 1. Características de recién nacidos con retinopatía del prematuro

\begin{tabular}{|c|c|c|c|c|c|}
\hline \multirow[t]{2}{*}{ Variable } & \multicolumn{2}{|c|}{ Con ROP $(n=156)$} & \multicolumn{2}{|c|}{ Sin ROP $(n=170)$} & $\mathbf{p}^{*}$ \\
\hline & \multicolumn{2}{|c|}{ Mediana (intervalo) } & \multicolumn{2}{|c|}{ Mediana (Intervalo) } & \\
\hline Edad gestacional & \multicolumn{2}{|c|}{$28(24-34)$} & \multicolumn{2}{|c|}{$30(23-35)$} & $<0.0001$ \\
\hline Peso al nacer (g) & \multicolumn{2}{|c|}{$1000(465-2290)$} & \multicolumn{2}{|c|}{$1260(370-1775)$} & $<0.0001$ \\
\hline Apgar al minuto 1 & \multicolumn{2}{|c|}{$6(0-8)$} & \multicolumn{2}{|c|}{$6(2-9)$} & ns \\
\hline Apgar al minuto 5 & \multicolumn{2}{|c|}{$7(2-9)$} & \multicolumn{2}{|c|}{$8(2-9)$} & ns \\
\hline Edad al ingreso (días) & \multicolumn{2}{|c|}{$47(1-154)$} & \multicolumn{2}{|c|}{$35(1-150)$} & $<0.0001$ \\
\hline Edad cronológica a la exploración oftalmológica (semanas) & \multicolumn{2}{|c|}{$8(2-22)$} & \multicolumn{2}{|c|}{$6(3-21)$} & $<0.001$ \\
\hline EPC a la exploración (semanas) & \multicolumn{2}{|c|}{$36(30-52)$} & \multicolumn{2}{|c|}{$36(30-51)$} & ns \\
\hline \multirow[t]{2}{*}{ Tiempo de uso de $\mathrm{O}_{2}$ (días) } & \multicolumn{2}{|c|}{$48(2-135)$} & \multicolumn{2}{|c|}{$35(3-98)$} & $<0.0001$ \\
\hline & $\mathrm{n}$ & $\%$ & $\mathrm{n}$ & $\%$ & \\
\hline \multicolumn{6}{|l|}{ Sexo } \\
\hline Masculino & 80 & 76 & 97 & 57.1 & $n s^{\star *}$ \\
\hline Femenino & 51.3 & 48.7 & 73 & 42.9 & \\
\hline \multicolumn{6}{|l|}{ Vía de nacimiento } \\
\hline Vaginal & 29 & 18.6 & 36 & 21.2 & $\mathrm{~ns}^{* *}$ \\
\hline Cesárea & 127 & 81.4 & 134 & 78.8 & \\
\hline Esteroide prenatal & 102 & 65.4 & 110 & 64.7 & $\mathrm{~ns}^{\star *}$ \\
\hline Surfactante & 147 & 94.2 & 139 & 81.8 & $0.001^{* *}$ \\
\hline
\end{tabular}

de la unidad hospitalaria. Dos investigadores recabaron la información de la primera evaluación y del seguimiento a partir de los expedientes clínicos.

\section{Análisis estadístico}

Se usó estadística descriptiva; para las variables cualitativas se calcularon frecuencias simples y porcentajes y para las cuantitativas, medianas e intervalos debido a que la mayoría de las variables no siguieron una distribución semejante a la normal.

Para el análisis comparativo se formaron dos grupos: niños con y sin ROP. Para la comparación de algunas variables se usó chi cuadrada o prueba exacta de Fisher para las variables cualitativas y $U$ de Mann-Whitney para las cuantitativas.

Para la elaboración de la base de datos y el análisis estadístico se empleó el programa estadístico SPSS versión 17.

\section{Resultados}

Se incluyeron $326 \mathrm{RN}$ entre enero de 2009 y diciembre de 2013: 156 niños con ROP y 170 sin ROP.
En la Tabla 1 se detallan las características generales de los RN de cada grupo; tanto la edad gestacional como el peso al nacer fue menor en los niños con ROP. El tiempo de uso de oxígeno fue mayor en los niños con ROP ( $p=0.0001)$. El surfactante se usó con mayor frecuencia en los $\mathrm{RN}$ que desarrollaron ROP ( $p=0.001)$. No hubo diferencia entre los grupos en la edad posconcepcional al momento de la exploración oftalmológica, sexo, vía de nacimiento ni administración de esteroide prenatal.

En la Tabla 2 se describe la edad cronológica y la edad posconcepcional en que los RN fueron evaluados por primera vez. También se muestra la recomendación internacional del momento ideal para realizar el primer examen oftalmológico.

A $73 \%$ de los niños que no desarrollaron ROP se les realizó la primera evaluación oftalmológica entre las cuatro y seis semanas de edad cronológica. Del total de pacientes con ROP, $40.4 \%$ fue examinado entre las cuatro y seis semanas de edad cronológica y solo $4.4 \%$ de los niños con ROP grave fueron evaluados a esa edad.

La mayoría de los RN tuvo más de una enfermedad asociada. La displasia broncopulmonar, el conducto 
Tabla 2. Relación entre la edad gestacional al nacimiento y la edad a la exploración oftalmológica

\begin{tabular}{|c|c|c|c|c|}
\hline \multirow[t]{2}{*}{$\begin{array}{l}\text { Edad gestacional } \\
\text { al nacimiento, semanas }\end{array}$} & \multicolumn{2}{|c|}{$\begin{array}{c}\text { Recomendación Academia Americana } \\
\text { de Pediatría }{ }^{22} \\
\text { Edad al primer examen (semanas)* }\end{array}$} & \multicolumn{2}{|c|}{$\begin{array}{c}\text { HP CMN SXXI } \\
\text { Edad al primer examen (semanas)* }\end{array}$} \\
\hline & Posconcepcional & Cronológica & Posconcepcional & Cronológica \\
\hline $22^{£}(0)$ & 31 & 9 & - & - \\
\hline $23^{\varepsilon}(1)$ & 31 & 8 & 28 & 5 \\
\hline $24(1)$ & 31 & 7 & 36 & 12 \\
\hline $25(4)$ & 31 & 6 & $31-41$ & $6-16$ \\
\hline $26(22)$ & 31 & 5 & $30-43$ & $4-17$ \\
\hline $27(41)$ & 31 & 4 & $31-44$ & $4-17$ \\
\hline $28(62)$ & 32 & 4 & $32-45$ & $4-17$ \\
\hline $29(38)$ & 33 & 4 & $33-44$ & $4-13$ \\
\hline $30(70)$ & 34 & 4 & $34-52$ & $4-22$ \\
\hline $31^{\ddagger}(11)$ & 35 & 4 & $34-39$ & $3-8$ \\
\hline $32^{\ddagger}(50)$ & 36 & 4 & $36-42$ & $4-10$ \\
\hline $33(12)$ & - & - & $37-42$ & $4-9$ \\
\hline $34(11)$ & - & - & $38-41$ & $4-7$ \\
\hline 35 (3) & - & - & $39-40$ & $4-5$ \\
\hline
\end{tabular}

*Recomendación en Estados Unidos.

**Hospital donde se llevó a cabo el estudio.

EEsta recomendación debe ser considerada tentativa más que basada en evidencia debido al pequeño número de sobrevivientes a esta edad.

†Si es necesario.

arterioso permeable, la hemorragia intraventricular y las crisis convulsivas fueron más frecuentes en los niños con ROP $(p<0.05)$.

La frecuencia de ROP fue de $47.8 \%(n=156 / 326)$. En 145 pacientes la ROP se detectó en ambos ojos $y$ en 11 fue unilateral.

Respecto a la gravedad, $79.9 \%$ tuvo ROP leve, $21.1 \%$ grave y $27.5 \%$ enfermedad plus. En la Tabla 3 se describe el estadio de la enfermedad y la zona afectada; la mayoría de los pacientes tuvo ROP en estadios 1 y 2 y en las zonas II y III; ninguno tuvo ROP en estadios 4 y 5 .

En la Figura 1 se aprecia la relación entre la edad gestacional y la frecuencia y gravedad de la ROP. La mayor frecuencia (27.6\%) se presentó en los RN con edad gestacional entre 27 y 29 semanas; en este grupo de edad también fue mayor la frecuencia de ROP grave (63.6 \%).

En relación con el peso al nacimiento, la ROP fue más frecuente en los que pesaron entre 751 y $1000 \mathrm{~g}$ (19\%), en esa categoría de peso también fue mayor la proporción de ROP grave (60.6 \%) (Figura 2).

Los pacientes que desarrollaron ROP tuvieron mayor duración de aporte de oxígeno, sin embargo, no se
Tabla 3. Frecuencia de retinopatía del prematuro de acuerdo con la clasificación internacional

\begin{tabular}{|c|c|c|c|c|c|c|c|c|}
\hline \multirow[t]{3}{*}{ Estadio } & \multicolumn{6}{|c|}{ Zona } & \multicolumn{2}{|c|}{ Total } \\
\hline & \multicolumn{2}{|c|}{ I } & \multicolumn{2}{|r|}{ II } & \multicolumn{2}{|c|}{ III } & \multirow[b]{2}{*}{$\mathrm{n}$} & \multirow[b]{2}{*}{$\%$} \\
\hline & n & $\%$ & $\mathrm{n}$ & $\%$ & $\mathrm{n}$ & $\%$ & & \\
\hline 1 & 2 & 1.3 & 15 & 9.6 & 63 & 40.4 & 80 & 51.3 \\
\hline 2 & 1 & 0.6 & 20 & 12.8 & 22 & 14.1 & 43 & 27.6 \\
\hline 3 & 2 & 1.3 & 16 & 10.3 & 15 & 9.6 & 33 & 21.1 \\
\hline Total & 5 & 3.2 & 51 & 32.7 & 100 & 64.1 & 156 & 100 \\
\hline \multicolumn{9}{|c|}{ Enfermedad plus } \\
\hline Estadio I & 0 & 0 & 4 & 2.5 & 1 & 0.6 & 5 & 3.2 \\
\hline Estadio II & 0 & 0 & 7 & 4.5 & 8 & 5.1 & 15 & 9.6 \\
\hline Estadio III & 2 & 1.3 & 13 & 8.3 & 8 & 5.1 & 23 & 14.7 \\
\hline Total & 2 & 1.3 & 24 & 5.3 & 17 & 10.8 & 43 & 27.5 \\
\hline
\end{tabular}

Once pacientes desarrollaron retinopatía del prematuro en un solo ojo.

pudo determinar las saturaciones de oxígeno que se manejaron en estos pacientes debido a que todos fueron referidos de otras unidades hospitalarias (Figura 3).

El $71.1 \%(111 / 156)$ de los RN con ROP recibió tratamiento, $63.4 \%$ con ROP leve y $100 \%$ con ROP grave. En $43.9 \%$ (54/123) de los niños con ROP leve 


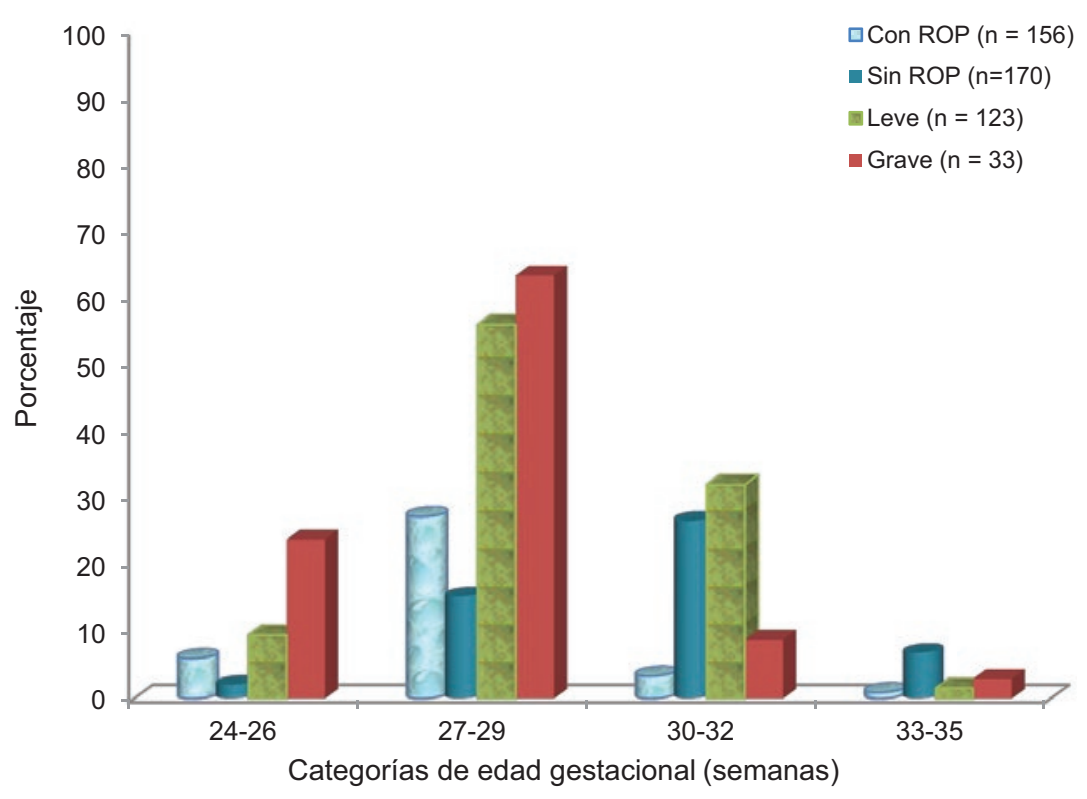

Figura 1. Frecuencia y gravedad de la retinopatía del prematuro (ROP) de acuerdo con la edad gestacional.

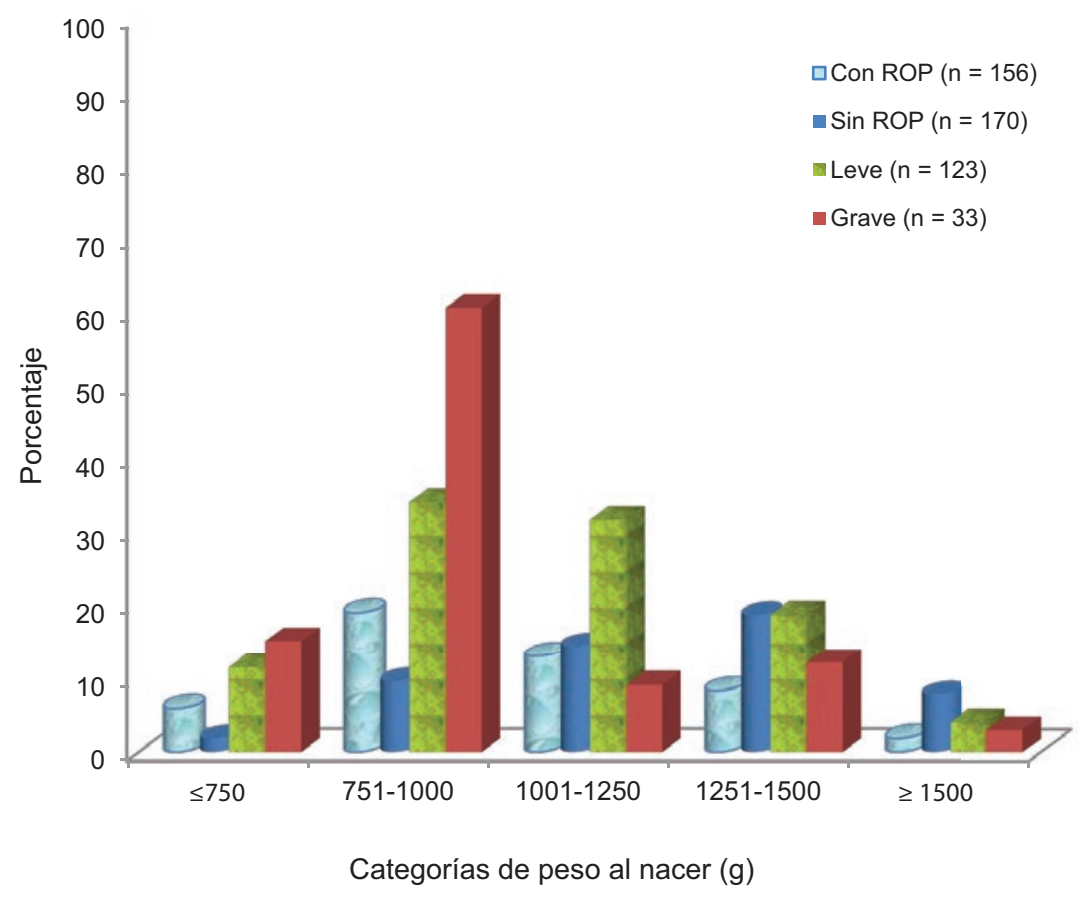

Figura 2. Frecuencia y gravedad de la retinopatía del prematuro $(R O P)$ de acuerdo con el peso al nacer.

se administró solo dexametasona paraocular (100 $\mu \mathrm{g} / \mathrm{kg} / \mathrm{dosis})$, en $19.5 \%(24 / 123)$ crioterapia, fotocoagulación con láser o una combinación de ellos, además, en 21 de estos RN se usó esteroide paraocular. En $36.4 \%$ (12/33) de los RN con ROP grave se administró esteroide y crioterapia; en $27.3 \%(9 / 33)$ crioterapia, en $27.3 \%$ (9/33) láser y esteroide paraocular y en $9 \%(3 / 33)$ solo láser.
Del total de pacientes incluidos, a 67 (20.5\%) se les realizó una segunda evaluación. El intervalo entre las dos evaluaciones tuvo una mediana de 16 días (rango de cinco a 69 días). De estos niños, 23 (34.3\%) tuvieron progresión de la ROP, 25 (37.3\%) regresión y $19(28.4 \%)$ se encontraron igual. De los 15 pacientes que en la evaluación inicial tuvieron inmadurez retiniana, en la segunda evaluación nueve habían 


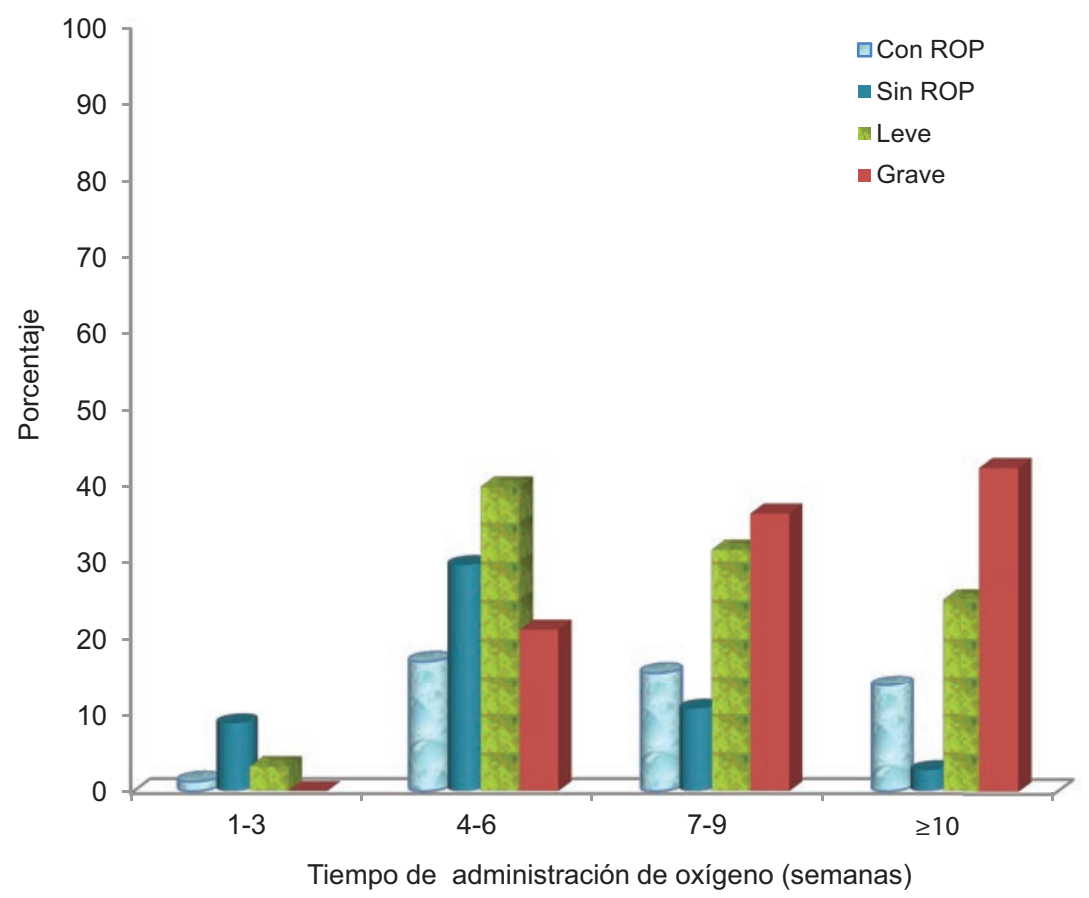

Figura 3. Frecuencia y gravedad de la retinopatía del prematuro (ROP) según el tiempo de uso de oxígeno suplementario.

progresado a ROP, tres mejoraron y tres permanecieron igual, es decir, con inmadurez.

\section{Discusión}

La ROP es una alteración del desarrollo de la retina en los RN prematuros de bajo peso al nacimiento que potencialmente produce ceguera, en un porcentaje bajo pero significativo. En los niños nacidos a término, la retina está completamente desarrollada, por lo que en ellos no ocurre ROP; sin embargo, en los RN prematuros, el desarrollo de la retina es incompleto y el grado de inmadurez de la retina depende principalmente del grado de prematurez del RN. ${ }^{22}$

La frecuencia de ROP en este estudio fue alta comparada con lo que se informa en algunos países desarrollados ${ }^{12,14,16}$ (aunque la red neonatal VOND reportó $57 \%){ }_{1}^{13}$ si bien está dentro del rango reportado en los países latinoamericanos, que oscila entre 6.6 y $82 \% ;{ }^{27}$ también fue similar a la de países de escaso desarrollo como Arabia Saudita, donde se reporta $56 \%{ }^{28}$ En México son escasos los registros de frecuencia de ROP, además de que los criterios de inclusión son diversos. ${ }^{18-21}$

El riesgo de desarrollar ROP no solo está determinado por la prematurez y el peso bajo al nacer, sino por otros factores como la gravedad del paciente durante su estancia en la UCIN, la comorbilidad y los manejos que reciben en ella, es decir, los pacientes que se manejan en cada unidad hospitalaria son diferentes, lo que hace difícil comparar las incidencias entre las distintas instituciones y más aún entre los diferentes países, sobre todo los desarrollados, donde se tienen bien establecidas políticas de detección de ROP. ${ }^{18,20,21}$

La frecuencia de ROP grave fue de $21.1 \%$, también mayor a la reportada. ${ }^{29}$ En España se registró una incidencia de $15.6 \%{ }^{17}$ y en Holanda de $1.2 \% .^{11}$ En este estudio fue similar a la registrada en los países latinoamericanos, cuya cifra se encuentra entre 1.2 y $23.8 \% .{ }^{27}$

De los factores que incrementan el riesgo para desarrollar ROP se encuentra indudablemente la edad gestacional y el peso al nacer. En el estudio ETROP ${ }^{12}$ se reportó una incidencia de $68 \%$ de ROP en los menores de $1251 \mathrm{~g}$. En Estados Unidos ${ }^{30}$ se encontró una incidencia de $30.9 \%$ en los niños con peso entre 750 y 999 g. En los RN analizados en el presente estudio se encontró una frecuencia de $37.7 \%$ en los niños con peso $\leq 1250 \mathrm{~g}$ y de $45.9 \%$ con peso $\leq 1500 \mathrm{~g}$, menor a lo informado en la India. ${ }^{31}$

Otro factor que se ha relacionado estrechamente con el desarrollo de ROP es el uso de oxígeno. Sin embargo, esto sigue siendo controversial: en algunos estudios se ha encontrado mayor incidencia de ROP con el uso indiscriminado de oxígeno a concentraciones altas, en otros no se ha demostrado esta asociación. ${ }^{32-40}$ De cualquier manera se recomienda el uso 
racional de oxígeno en todos los RN prematuros, principalmente en los más inmaduros. Por su parte, Chow $^{26}$ propone que en los RN prematuros deben mantenerse saturaciones de oxígeno similares a las fetales y nunca superiores a $95 \%$. En la presente investigación se encontró que a mayor tiempo de uso de oxígeno, mayor frecuencia de ROP, sin embargo, lo más importante es la saturación de oxígeno que alcanzan los pacientes, información que no fue posible obtener en esta investigación dado el diseño del estudio y las características del hospital donde se realizó, ya que los pacientes que ingresan a la UCIN son referidos de otros hospitales con un resumen clínico donde no se anotan las concentraciones de oxígeno empleadas ni las saturaciones alcanzadas, solo se indica el tiempo de uso.

Todos los pacientes tuvieron una o más comorbilidades, con una diferencia estadísticamente significativa en displasia broncopulmonar y hemorragia intraventricular, observándose con mayor frecuencia en los niños con ROP, similar a lo reportado por Lad..$^{30}$ También se encontró que el conducto arterioso permeable y las crisis convulsivas fueron más frecuentes en ellos, con una diferencia estadísticamente significativa.

Debido a que la naturaleza de la ROP es la progresión y que el tratamiento oportuno reduce el riesgo de pérdida visual, se requiere que los $\mathrm{RN}$ prematuros con alto riesgo reciban un cuidadoso examen por un oftalmólogo experimentado y que todos los pediatras y neonatólogos encargados del cuidado de estos prematuros tengan conocimiento de las implicaciones de esta patología.

En países desarrollados como Estados Unidos y el Reino Unido se han establecido guías para el tamizaje de estos niños, pero es importante tener en cuenta que cada región puede tener diferentes parámetros al respecto, debido a que las características de los pacientes y los manejos en las UCIN son diferentes. ${ }^{22}$ En esas guías se recomienda el tamizaje para los niños $\leq 32$ semanas de gestación o con peso al nacer < $1500 \mathrm{~g}$; en México, el lineamiento técnico recomienda el tamizaje en los $\mathrm{RN}$ con edad gestacional $\leq 34$ semanas y peso al nacer $\leq 1750 \mathrm{~g} .{ }^{41}$

Se encontró que en los niños analizados la primera evaluación se realizó tardíamente según las recomendaciones internacionales. De acuerdo con la recomendación de la Academia Americana de Pediatría sección Oftalmología, ${ }^{22}$ los niños con edad gestacional de 27 semanas deberían ser evaluados a las 31 semanas de edad posconcepcional o a las cuatro semanas de edad cronológica; en nuestro estudio, los niños de esa edad fueron revisados entre las 31 y 44 semanas de edad posconcepcional y entre las cuatro y 17 semanas de edad cronológica.

A muy pocos pacientes $(20.5 \%)$ incluidos en el presente estudio se les realizó una segunda evaluación en el mismo hospital y el intervalo entre una y otra fue largo. Lo anterior pudiera deberse a la falta de recursos para enviar a los pacientes al centro de referencia, además de que en los hospitales generales o regionales de zona no hay recursos humanos ni tecnológicos para la atención de estos niños, es decir, para establecer diagnóstico y tratamiento oportunos y adecuados, así como el seguimiento y la rehabilitación necesarios. En una encuesta realizada a pediatras y neonatólogos, Zepeda et al..$^{42}$ encontraron que las principales barreras para establecer programas de detección y tratamiento efectivo son la falta de recursos humanos, la falta de acceso a la tecnología y de equipo adecuado, la falta de recursos económicos y de apoyo institucional, así como la falta de conocimiento de la enfermedad.

Respecto al tratamiento, una modalidad instituida en este hospital es la aplicación de dexametasona paraocular cuando se detecta ROP en estadios leves y después raramente se requieren tratamientos más avanzados como crioterapia o láser. Es posible que por sus propiedades antiinflamatorias el esteroide actúe como antiangiogénico. Este tratamiento no está referido en la literatura, por lo que queda por analizar la evolución a corto, mediano y largo plazo de los niños a quienes se administra dexametasona paraocular.

Debido al incremento en la supervivencia de los RN prematuros se espera que la incidencia de ROP se mantenga elevada, por lo que consideramos necesario realizar estudios para identificar la frecuencia de ROP en México para establecer programas de detección oportuna y así proporcionarles el tratamiento y seguimiento correspondientes.

\section{Bibliografía}

1. Flynn J. Retinopathy of prematurity. En: Eichenbaum JW. Treatment of retinopathy of prematurity. EE. UU.: Year Book Medical Publishers; 1990.

2. Terry TL. Extreme prematurity and fibroblastic overgrowth of persistent vascular sheath behind each crystalline lens: I. Preliminary report. Am J Opthalmol. 2018;192:xxviii.

3. Heath P. Pathology of the retinopathy of prematurity: retrolental fibroplasia. Am J Ophthalmol. 1951;34:1249-1259.

4. Gilbert C, Fielder A, Gordillo L, Quinn G, Semiglia R, Visintin P, et al. Characteristics of infants with severe retinopathy of prematurity in countries with low, moderate and high levels of development: Implication for screening programs. Pediatrics. 2005;115(5):e518-e525.

5. Manual of operations. Release 13.2. EE. UU.: Vermont Oxford Network; 2009

6. Kinsey VE, Hemphill FM. Etiology of retrolental fibroplasia and preliminary report of cooperative study of retrolental fibroplasia. Trans Am Acad Ophthalmol Otolaryngol. 1955:59:15-24.

7. Gibson DL, Sheps SB, Uh SH, Schechter MT, McCormick AQ. Retinopathy of prematurity-induced blindness: birth weight-specific survival and the new epidemic. Pediatrics. 1990;86:405-412. 
8. Kinsey VE. Retrolental fibroplasia; cooperative study of retrolental fibroplasia and the use of oxygen. AMA Arch Ophthalmol. 1956;56:481-543.

9. Palmer EA, Flynn JT, Hardy RJ, Phelps DL, Phillips CL, Schaffer DB, et al. Incidence and early course of retinopathy of prematurity. The Cryotherapy for Retinopathy of Prematurity Cooperative Group. Ophthalmology. 1991;98:1628-1640.

10. Bullard SR, Donahue SP, Feman SS, Sinatra RB, Walsh WF. The decreasing incidence and severity of retinopathy of prematurity. J AAPOS 1999;3:46-52.

11. Good WV, Hardy RJ, Dobson V, Palmer EA, Phelps DL, Quintos M, et al. The incidence and course of retinopathy of prematurity: findings from the early treatment for retinopathy of prematurity study. Pediatrics. 2005;116:15-23.

12. Hoogerwerf A, Schalij-Delfos NE, Van-Schooneveld MJ, Termote JU. Incidence of retinopathy of prematurity over the last decade in the Central Netherlands. Neonatology. 2010;98:137-142.

13. Quinn GE, Barr C, Bremer D, Fellows R, Gong A, Hoffman R, et al. Changes in course of retinopathy of prematurity from 1986 to 2013: comparison of three studies in the United States. Ophthalmology. 2016; 123:1595-1600.

14. Gerull R, Brauer V, Bassler D, Laubscher B, Nelle M, Müller B, et al. Incidence of retinopathy of prematurity (ROP) and ROP treatment in Switzerland 2006-2015: a population-based analysis. Arch Dis Child Fetal Neonatal Ed. 2018;103:F337-F342.

15. Ludwig CA, Chen TA, Hernandez-Boussard T, Moshfeghi AA, Moshfeghi DM. The epidemiology of retinopathy of prematurity in the United States. Ophthalmic Surg Lasers Imaging Retina. 2017;48:553-562.

16. Park SH, Yum HR, Kim S, Lee YC. Retinopathy of prematurity in Korean infants with birthweight greater than $1500 \mathrm{~g}$. Br J Ophthalmol. 2016 100:834-838.

17. Hernández M, Orduna C, Bosch V, Salinas R, Alcaraz JL, Marín JM. Retinopatía del prematuro en la región de Murcia (España). Incidencia y gravedad. Arch Soc Esp Oftalmol. 2008;83:423-428.

18. Cervantes-Munguía R, Espinosa-López L, Gómez-Contreras $P$, Hernández-Flores G, Domínguez-Rodríguez J, Bravo-Cuéllar A. Retinopatía del prematuro y estrés oxidativo. An Pediatr (Barc). 2006;64:126-131.

19. González-Urquidi O, De la-Fuente-Torres M. Incidencia de retinopatía del prematuro en el hospital Dr. Manuel Gea González. Rev Mex Oftalmol. 2004;78:1-4

20. Sánchez E, Zapata G, Escamilla M. Retinopatía en el prematuro menor a $1500 \mathrm{gr}$ expuesto a concentraciones altas de oxígeno suplementario. Incidencia en Yucatán. Rev Mex Oftalmol. 2008;82:381-384.

21. Orozco-Gómez P, Ruiz-Morfin I, Lámbarry-Arroyo A, Morales-Cruz MV Prevalencia de retinopatía del prematuro. 12 años de detección en el Centro Médico 20 de noviembre. Cir Ciruj. 2006;74:3-9.

22. Section on Ophthalmology American Academy of Pediatrics; American Academy of Ophthalmology; American Association for Pediatric Ophthalmology and Strabismus. Screening examination of premature infants for retinopathy of prematurity. Pediatrics. 2006;117:572-576.

23. Good WV, Early Treatment for Retinopathy of Prematurity Cooperative Group. The Early Treatment for Retinopathy of Prematurity Study; structural findings at age 2 years. Br J Ophthalmol. 2006;90:1378-1382.

24. Msall ME, Phelps DL, Hardy RJ, Dobson V, Quinn GE, Summers CG, et al. Educational and social competencies at 8 years in children with threshold retinopathy of prematurity in the CRYO-ROP multicenter study. Pediatrics. 2004;113:790-799.
25. International Committee for the Classification of Retinopathy of Prematurity. The International Classification of Retinopathy of Prematurity revisited. Arch Ophthalmol. 2005;123:991-999.

26. Chow LC, Wright KW, Sola A; CSMC Oxygen Administration Study Group. Can changes in clinical practice decrease the incidence of severe retinopathy of prematurity in very low birth weight infants? Pediatrics. 2003;111:339-345.

27. Zimmermann-Carrion J, Fortes-Filho FJB, Tartarella MB, Zin A, Dorneles-Jornada I. Prevalence of retinopathy of prematurity in Latin America. Clin Ophthalmol. 2011;5:1687-1695.

28. Binkhathlan AA, Almahmoud LA, Saleh MJ, Srungeri S. Retinopathy of prematurity in Saudi Arabia incidence, risk factors, and applicability of current screening criteria. Br J Ophthalmol. 2008;92:167-169.

29. Hameed B, Shyamanur K, Kotecha S, Manktelow B, Woodruff G, Draper ES, et al. Trends in the incidence of severe retinopathy of prematurity in a geographically defined population over a 10-year period. Pediatrics. 2004;113:1653-1657.

30. Lad EM, Hernández-Boussard T, Morton JM, Moshfeghi DM. Incidence of retinopathy of prematurity in the United States: 1997 through 2005. Am J Ophthalmol. 2009;148:451-458.

31. Vinekar A, Dogra MR, Sangtam T, Narang A, Gupta A. Retinopathy of prematurity in Asian Indian babies weighing greater than 1250 grams at birth: ten year data from a tertiary care center in a developing country. Indian J Ophthalmol. 2007;55:331-336.

32. Sola A, Chow L, Rogido M. Retinopathy of prematurity and oxygen therapy: A changing relationship. An Pediatr (Barc). 2005;62:48-63.

33. Carlo WA, Finer NN, Walsh MC, Rich W, Gantz MG, Laptook AR, et al. Target ranges of oxygen saturation in extremely preterm infants. $\mathrm{N}$ Engl J Med. 2010;362:1959-1969.

34. Bedrossian $\mathrm{RH}$, Carmichael P, Ritter JA. Effect of oxygen weaning in retrolental fibroplasia. AMA Arch Ophthalmol. 1954;53:514-518.

35. Bedrossian $\mathrm{RH}$, Carmichael $\mathrm{P}$, Ritter J. Retinopathy of prematurity (retrolental fibroplasia) and oxygen. I. Clinical study. II. Further observations on the disease. Am J Ophthalmol. 1954;37:78-86.

36. Hittner HM, Speer ME, Rudolph AJ, Blifeld C, Chadda P, Holbein ME, et al. Retrolental fibroplasia and vitamin $\mathrm{E}$ in the preterm infant - comparison of oral versus intramuscular: oral administration. Pediatrics. 1984;73:238-249.

37. Gaynon MW, Stevenson DK, Sunshine P, Fleisher BE, Landers MB. Supplemental oxygen may decrease progression of prethreshold disease to threshold retinopathy of prematurity. J Perinatol 1997;17:434-438.

38. Lloyd J, Askie L, Smith J, Tarnow-Mordi W. Supplemental oxygen for the treatment of prethreshold retinopathy of prematurity. Cochrane Database Syst Rev. 2003;2:CD003482.

39. Tin W, Milligan DW, Pennefather P, Hay E. Pulse oximetry, severe retinopathy, and outcome at one year in babies of less than 28 weeks gestation. Arch Dis Child Fetal Neonatal Ed. 2001;84:106-110.

40. Chen ML, Guo L, Smith LE, Dammann CE, Dammann O. High or low oxygen saturation and severe retinopathy of prematurity: a meta-analysis. Pediatrics. 2010;125:1483-1492.

41. Secretaría de Salud. Manejo de la retinopatía del recién nacido prematuro. Lineamiento técnico. México: Secretaría de Salud; 2007.

42. Zepeda-Romero LC, Gutiérrez-Padilla JA, De la Fuente-Torres MA, Angulo-Castellanos E, Ramos-Padilla E, Quinn GE. Detection and treatment for retinopathy of prematurity in Mexico: need for effective programs. J AAPOS. 2008;12:225-226. 